\title{
The perception of shape from shading in a new light
}

How do humans see three-dimensional shape based on two-dimensional shading? Much research has assumed that a 'light from above' bias solves the ambiguity of shape from shading. Counter to the 'light from above' bias, studies of Bayesian priors have found that such a bias can be swayed by other light cues. Despite the persuasive power of the Bayesian models, many new studies and books cite the original 'light from above' findings. Here I present a version of the Bayesian result that can be experienced. The perception of shapefrom-shading based was found here to be influenced by an external light source, even when the light was obstructed and did not directly illuminate a two-dimensional stimulus. The results imply that this effect is robust and not low-level in nature. The perception of shape from shading is not necessarily based on a hard-wired internal representation of lighting direction, but rather assesses the direction of lighting in the scene adaptively. Here, for the first time, is an experiential opportunity to see what the Bayesian models have supported all along. 
8 Correspondence should be addressed to

9 Department of Psychology

102 South

11 University of Bath

12 Bath, BA2 7AY, UK

E-mail: mproulxbath@gmail.com research has assumed that a 'light from above' bias solves the ambiguity of shape from shading.

17 Counter to the 'light from above' bias, studies of Bayesian priors have found that such a bias can

18 be swayed by other light cues. Despite the persuasive power of the Bayesian models, many new

19 studies and books cite the original 'light from above' findings. Here I present a version of the

20 Bayesian result that can be experienced. The perception of shape-from-shading based was found

21 here to be influenced by an external light source, even when the light was obstructed and did not

22 directly illuminate a two-dimensional stimulus. The results imply that this effect is robust and not

23 low-level in nature. The perception of shape from shading is not necessarily based on a hard-

24 wired internal representation of lighting direction, but rather assesses the direction of lighting in

25 the scene adaptively. Here, for the first time, is an experiential opportunity to see what the

26 Bayesian models have supported all along. 


\title{
28 Introduction
}

29

\begin{abstract}
A person entering into a room perceives, at a single glance, whence the light comes which illuminates the objects before him; and that without remaining conscious for a moment that he has attended to the circumstance: But the effect remains, and will influence his judgment. [pp 38-39]
\end{abstract}

A number of fascinating illusions arise from graded changes in lumination. Some of the most striking give rise to the appearance of light emitting from a constant image, such as the glare effect or the breathing light illusion .A careful arrangement of light and shadow, or a steady change in luminance, can mimic natural light in surprising ways and even represent threedimensional shape based on shading cues alone. For example, how does one see threedimensional shape based on two-dimensional shading, such as that shown in Figure 1A? Initial research found that the brain assumes a single 'light from above' illuminating the discs , suggesting that the brain is hard-wired by evolution to assume a light source that is overhead like the sun . Turning the figure by 90 degrees weakens the 3-D impression. More recent research found that not only is there a light from above, and to the left . Surprisingly, research with chimpanzees revealed an assumption of a light source coming from the side; unlike humans, turning the figure by 90 degrees strengthens the 3-D percept in chimpanzees . Have humans and chimpanzees evolved different neural assumptions about an inferred light location? The 'single light from above' hypothesis is the most favoured perspective amongst researchers in recent publications . Relying instead on an external light source would make adaptive sense: rather than having an assumption that might come into conflict with an actual source of object shading, one should take advantage of an external light cue when interpreting scenes. Any conflict between the actual light source and that perceived in computer graphics could slow responses and reduce accuracy. 
Although most recent investigators have accepted the "light is overhead" bias as a strong

56 rule used by the human visual system, A number of studies have taken a Bayesian modelling

57 approach to demonstrate how other information is incorporated with such a prior to modify the

58 ultimate perception in favour of the most likely outcome. Contrary to those who theorized that

59 the overhead bias is hard-wired and innate, Stone found that the overhead Bayesian prior

60 requires developmental experience. A recent study demonstrated that the assumption that light

61 comes from above has a lesser role than lighting cues in the perception of shape from shading .

62 Morgenstern and colleagues presented lighting cues that could influence shape perception in

63 either accordance with an objects context in the image or instead with the light from above prior.

64 The light direction implied by the context had a greater effect on shape from shading perception.

65 Also, little notice has been given to aspects of previous research that suggest the overhead bias

66 can be overridden by an external light source. Yonas et al have most often been cited for

67 demonstrating that children primarily perceived shape from shading in egocentric "light is

68 overhead" coordinates. However, they also reported that a significant proportion of the subjects

69 perceived the stimuli in reference to actual external light as well (though at a lower proportion

70 than those using the egocentric reference frame). What is remarkable about these results is that

71 even though the light source was covered (thus perhaps making its location ambiguous), a

72 significant number of the children perceived the stimuli as shaded consistent with the light's

73 location. This also points to possible individual differences in a seemingly simple, low-level task,

74 has others have noted as well.

75 Berbaum and colleagues examined the impact of the direction of illumination on the

76 visual interpretation of an actual, three-dimensional muffin pan. Subjects viewed the pan with

77 the light coming from below; by incorporating mirrors, however, the experimenters had it appear

78 to the subjects that the light was actually coming from above. The subjects perceived the shape

79 based on the remembered light source direction (the light source could not actually be seen at the 
same time as the stimulus). The extension of this work to two-dimensional stimuli has been

81 questioned because it had not been replicated in previous work .

82 However, there are two inaccuracies to this criticism by Kleffner and Ramachandran .

83 First, the study by Berbaum et al was incorrectly cited as having had the subjects discover the

84 actual light source direction by putting out their hands to cast a shadow, with a resulting inversion

85 of relief when the light source direction was discovered. In fact, the Berbaum et al study put

86 sticks on the muffin pans to cast shadows that revealed the true direction of illumination and they

87 found that this did not cause a reversal of relief (the perception continued to be based on the

88 direction of remembered illumination direction). Second, the previous study by Ramachandran

89 has its own drawbacks as well in terms of addressing the impact of illumination direction. The

90 direction of illumination was itself ambiguous: a concave mask of a face, although lit from above,

91 is perceived as a normal face lit from below; thus the direction of illumination was assumed by

92 the experimenter to be from below (consistent with the face perception) however subjects still

93 viewed the shape from shading discs as if they were lit from above (consistent with the actual

94 direction of illumination on the convex mask that appeared as a concave face).

95 Here we tested a demonstration that the visual system might rely more on an external light

96 cue to judge shape from shading with a straightforward demonstration in the spirit of

97 Ramachandran . Given the evidence presented by a number of studies, summarized and tested by

98 Morgenstern and colleagues, it should be well known now that the original finding of a light

99 from overhead bias is not the whole story. Despite such evidence to the contrary, the light from

100 overhead view is still often cited. Here we found a robust version of the shape from shading

101 experiment that can be experienced even with a printed photograph of a shape from shading

102 stimulus and an external light cue, thus rendering the effect in manner suitable for a sceptical

103 reader or a classroom setting.

\section{Methods}




\section{Subjects}

104 Naive observers viewed stimuli from a distance of $50 \mathrm{~cm}$, and under only one of the three

105 conditions: overhead lighting $(n=5)$; a light below $(n=14)$; or an obstructed light below $(n=9)$.

106 All observers had normal or corrected-to-normal visual acuity (wearing glasses or contact lenses

107 if necessary). Ethical approval was obtained from the JHU IRB, QMREC and Bath Psychology

108 Ethics Committee, and all participants gave informed, written consent.

109 Stimuli \& Procedure

110 The reader can replicate the effect used here in a dark room with a single light placed

111 below the image in Figure 1A. The discs ( $2 \mathrm{~cm}$ in diameter) had a vertical luminance gradient

112 from white at one end to black at the other, with the centre of the disc at the same level of grey as

113 the background, and were printed on standard (flat) white paper.

114 In conditions with the light from below, the room was completely dark except for an

115 incandescent light (30W) placed below the stimuli, except for the condition where a thin board

116 obstructed the entire beam of light. The direction of the actual light source was a between-

117 subjects manipulation to avoid drawing attention to the light. The observers told the

118 experimenter whether the different discs appeared either as bumps or cavities, and were never

119 asked about any real or implied light source. Note that there were a total of 36 discs shown on

120 seven different trials each with a different configuration of 1 to 12 discs to each observer. The

121 observers were asked to point to each disc present for each trials and report if it appeared as a

122 bump or a cavity. All observers reported the discs as bumps or cavities in a manner consistent

123 with perceiving only one light source; that is, no participants ever reported discs with opposite

124 polarity of shading as both being bumps or both being cavities. Each configuration of discs was

125 shown to each participant once.

126 The stimuli were printed on paper so all competing light cues (including a back-lit

127 monitor) were removed and the shape from shading of the discs was completely ambiguous, 
128 unlike previous work. The responses for each disc were converted to light direction ratings to

129 reflect whether observers perceived a stimulus as being lit from above or from below. The light

130 direction was inferred from their judgments of disc convexity. For example, if a disc was labelled

131 a "bump" and its shading was white at the top, and black at the bottom, then this indicated that

132 the perceived direction of illumination was from above because a bump would be brightest at the

133 portion nearest the illumination. If the perceived direction of illumination was above, then the

134 response was recorded as " 1 "; if the perceived direction of illumination was below, then the

135 response was recorded as " 0 ". Thus, if all observers perceived every disc as being lit from

136 overhead, then the average rating of illumination direction would be 1 . Thus the analysis is

137 similar to that of assessing accuracy as a proportion, however here computed as a function of how

138 each disc was classified. All analyses were based on these ratings, averaged across observers and

139 trials. The null hypothesis on the basis of the literature and the overhead light condition is that all

140 ratings should be rated as 1.0, consistent with the 'light from above' assumption . To provide a

141 directional test of the hypothesis a one-tailed $t$-test was used to evaluate the results; however

142 exact $p$ values are reported also.

\section{Results}

$144 \quad$ Overhead light

145 In the overhead lighting condition, all participants reported all stimuli as bumps or

146 cavities in a manner consistent with the 'light from above' assumption, resulting in a rating of 1.0

147 (see Figure 1B, 'Overhead Light'), replicating the original finding .

$148 \quad$ Light below

149 Figure 1B shows that, when the external light source was below the images, the average

150 proportion was only 0.48 (see 'Light Below'); this indicates that the stimuli were often perceived

151 in a manner consistent with the external light and inconsistent with the 'light from above' 
152 assumption, $t(13)=-6.18, P=.00003, \eta^{2}=0.718$ (one-tailed $t$-test). Individual differences were

153 primarily responsible, with six out of 14 observers' responses consistent with the light source.

154 Two possible mechanisms might be responsible for the effect of the external light: first, a

155 low-level process whereby cues created by the external light interact with the grain of the paper

156 on which the stimuli were presented; or second, a higher level process, whereby a representation

157 of the physical location of the light source is responsible for the influence of an external light .

$158 \quad$ Obstructed light below

159 To distinguish between these mechanisms, a third condition featured a light source again

160 placed below the stimuli, however a board was placed between the light and the stimuli, and thus

161 the light was indirect. If the previous results were due to low-level shading cues, then the average

162 rating should be closer to 1.0, consistent with the 'light from above' assumption. In fact, when the

163 light was obstructed such that there were no low-level light cues on the stimulus (see 'Obstructed

164 Light Below'), the average rating was only 0.35 ; this means that stimuli were again perceived in

165 a manner consistent with the external light cue, $t(8)=-6.61, P=.0002, \eta^{2}=0.845$ (one-tailed $t$ -

166 test). Seven out of nine of the observers were primarily affected by the light source. There was

167 not a significant difference between the unobstructed and obstructed light below conditions.

\section{Discussion}

169 The results are inconsistent with the hypothesis that the visual system relies exclusively

170 on the 'light from above' assumption, and furthermore demonstrate that this effect is not low-

171 level in nature, consistent with past work on this topic that found a memorial representation of

172 light location was used to interpret the shape of an actual muffin tin even when the light was

173 occluded and did not directly illuminate a two-dimensional stimulus. Note that similar to

174 previous research by Yonas and colleagues with children, individual differences in the perception

175 of shape-from-shading are present, and future research will better reveal the source of this

176 diversity. The results further imply that this effect is not low-level in nature, and thus consistent 
177 with the quote from Rittenhouse's initial article the described shape from shading for the first

178 time .

179 The present findings provide converging support with recent behavioural evidence that

180 lighting cues, rather than a 'light from above' bias, are the primary determinant of the perception

181 of shape from shading. The results reported here provide further evidence that the perception of

182 shape from shading, like other aspects of visual perception, arises from interactions with the

183 natural world rather than internal biases that give rise to illusory perception . Importantly the

184 demonstration here does not depend on a complex analysis of psychophysical data, but rather a

185 simple shape-from-shading drawing and a candle would do, allowing even Rittenhouse to

186 experience this himself. 


\section{Acknowledgements}

I thank Christopher Min and Monique Green for assistance, and Howard Egeth for helpful discussions.

\section{Funding statement}

Supported by the US National Science Foundation (GRF). 


\section{Literature Cited}

187 [1] Rittenhouse D. Explanation of an optical deception. Transactions of the American

[18] Berbaum K, Bever T, Chung CS. Light source position in the perception of object shape. Perception 1983; 12: 411-416.

[19] Yonas A, Kuskowski M, Sternfels S. The role of frames of reference in the development of responsiveness to shading information. Child Dev 1979; 50: 495-500.

[20] Koenderink JJ, van Doorn AJ, Kappers AM. Surface perception in pictures. Perception and Psychophysics 1992; 52: 487-496.

[21] Todd JT, Koenderink JJ, van Doorn AJ, Kappers AM. Effects of changing viewing conditions on the perceived structure of smoothly curved surfaces. Journal of Experimental Psychology: Human Perception and Performance 1996; 22: 695-706.

[22] Kleffner DA, Ramachandran VS. On the perception of shape from shading. Perception and Psychophysics 1992; 52: 18-36.

[23] Kleffner DA, Ramachandran VS. On the perception of shape from shading. Percept Psychophys 1992; 52: 18-36.

[24] Wenderoth P, Hickey N. Object and head orientation effects on symmetry perception defined by shape from shading. Perception 1993; 22: 1121-1130. 
234 [25] Purves D, Wojtach WT, Lotto RB. Understanding vision in wholly empirical terms.

235

236

237

238

239

240 Proceedings of the National Academy of Sciences U S A 2011; 108 Suppl 3: 1558815595.

[26] Stone JV, Pascalis O. Footprints sticking out of the sand. Part 1: Children's perception of naturalistic and embossed symbol stimuli. Perception 2010; 39: 1254-1260. 


\section{Figure Title}

242 Figure 1. A. An example stimulus. B. Mean rating of perceived light direction versus the actual 243 illumination direction in the room, as a function of experimental condition (Condition 1:

244 Overhead Light; Condition 2: Light Below; Condition 3: Obstructed Light Below). 1.0 = light 245 from above; $0.0=$ light from below. Error bars are \pm 1 s.e.m.

246 圆 


\section{Figure 1}

Stimulus and results

Figure 1A. An example stimulus. 1B. Mean rating of perceived light direction versus the actual illumination direction in the room, as a function of experimental condition (Condition 1 :

Overhead Light; Condition 2: Light Below; Condition 3: Obstructed Light Below). 1.0 = light from above; 0.0 = light from below. Error bars are \pm 1 s.e.m. 


\section{A}
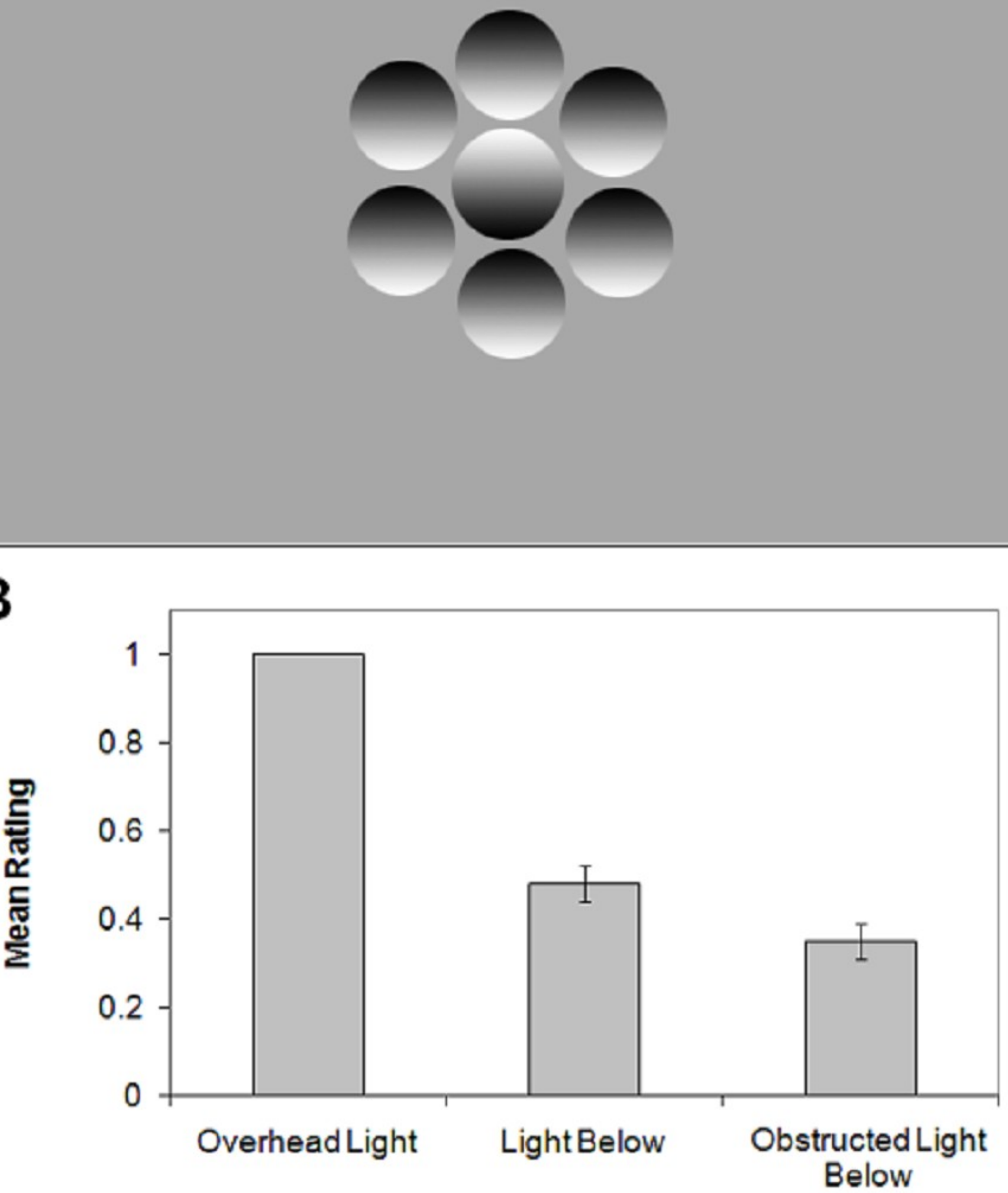

Actual Light Direction 